\title{
Bacteriemia por Vibrio cholerae no-O1/no-O139 que porta una región homóloga a la isla de patogenicidad VpaI-7
}

\author{
Felipe Olivares ${ }^{1}$, Ignacio Domínguez², Jeannette Dabanch ${ }^{3}$, Lorena Porte ${ }^{3,4}$, M. Teresa Ulloa ${ }^{5}$ y Gonzalo Osorio ${ }^{5}$
}

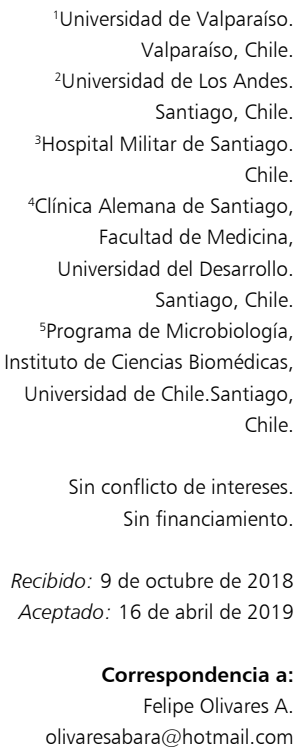

'Universidad de Valparaíso. Valparaíso, Chile. ¿Universidad de Los Andes. Santiago, Chile. ${ }^{3}$ Hospital Militar de Santiago. Chile. ${ }^{4}$ Clínica Alemana de Santiago, Facultad de Medicina, Universidad del Desarrollo. Santiago, Chile.

SPrograma de Microbiología, Instituto de Ciencias Biomédicas, Universidad de Chile.Santiago, Chile.

Sin conflicto de intereses. Sin financiamiento.

Recibido: 9 de octubre de 2018 Aceptado: 16 de abril de 2019

Correspondencia a: Felipe Olivares A. olivaresabara@hotmail.com

\section{Bacteremia caused by Vibrio cholerae non-O1/non-O139 carrying a region homologous to pathogenicity island VpaI-7}

We report a case of $V$. cholerae non-O1 / non-O139 bacteremia in an 81-year-old woman with abdominal pain, fever, vomiting, liquid stools, choluria and jaundice, while visiting a rural area without access to potable water. The identification was made by the MALDI-TOF mass spectrometry technique and subsequently the non-toxigenic non-O1 / non-139 strain was confirmed in the national reference laboratory. The molecular characterization demonstrated the absence of the cholera toxin gene (CTX), and the TCP pilus, however, presented 5 of 6 virulence genes present in an island of homologous pathogenicity named VPaI-7 of $V$. parahaemolyticus (vcs $\mathrm{N} 2+, \mathrm{vcs} C 2$ + , vcs $\mathrm{V} 2+$, toxR-, vspD +, T vopF +) and in addition it was positive for hylAy rtxA virulence genes recognized outside the island. This is the first case reported in Chile of a clinical strain of $V$. cholerae non-O1, non-O139 isolated from blood culture that carries in its genome a homologous segment of the pathogenicity island named VPaI-7 of V. parahaemolyticus, which codifies for a type III secretion system (TTSS) that probably contributes to his virulence.

Keywords: Bacteremia, Vibrio cholerae, non-O1/non-O139; pathogenicity island.

Palabras clave: Bacteriemia; Vibrio cholerae, no-O1/no-O139; isla de patogenicidad.

\section{Introducción}

V ibrio cholerae es un bacilo gramnegativo causante del cólera, un tipo de diarrea secretora que se ha asociado a grandes brotes epidémicos a lo largo de la historia, particularmente los serogrupos O-1 y O-139, los cuales tienen la capacidad de producir toxinas ${ }^{1-4}$.

Según el tipo de antígeno $\mathrm{O}$, se han descrito más de 200 serogrupos $^{5}$, aunque la mayoría no son clasificables y se les denomina como no-O1/no-O1396. Estas cepas se han asociado a cuadros gastrointestinales de menor magnitud; sin embargo, cada vez más se describen infecciones extra-intestinales, incluyendo bacteriemias. Esto ha generado el interés por identificar nuevos factores de virulencia presentes en este tipo de cepas, dado que no tienen la capacidad de producir toxinas ${ }^{7-10}$.

Se reporta un caso de bacteriemia por $V$. cholerae no-O1/no-O139 en el cual se realizó identificación microbiológica y caracterización molecular de posibles genes de virulencia.

\section{Caso clínico}

Mujer de 81 años, con antecedente de colecistectomía, que consultó por cuadro de una semana de evolución caracterizado por dolor abdominal epigástrico irradiado a hipocondrio derecho, fiebre, ictericia, coluria y diarrea de baja cuantía. La sintomatología se presentó durante su estadía en una zona rural sin acceso a agua potable. Al examen físico se encontraba afebril, con hemodinamia estable, escleras ictéricas, y sensibilidad aumentada a la palpación en hipocondrio derecho sin signos de irritación peritoneal. En los exámenes de laboratorio presentaba leucocitosis $\left(19.400 / \mathrm{mm}^{3}\right)$ con neutrofilia, PCR elevada $(197 \mathrm{mg} / \mathrm{L})$ y bilirrubina total alta $(3,5 \mathrm{mg} / \mathrm{dl})$. Las transaminasas estaban discretamente aumentadas (GOT $66 \mathrm{UI} / \mathrm{L}, \mathrm{GPT} 87 \mathrm{UI} / \mathrm{L}, \mathrm{GGT} 187 \mathrm{UI} / \mathrm{L}$ ), al igual que las fosfatasas alcalinas (220 UI/L). La ecografía abdominal mostró dilatación de la vía biliar extra-hepática hasta $7,8 \mathrm{~mm}$ y la colangioresonancia, una vía biliar fina sin colédocolitiasis y ausencia de otros hallazgos patológicos. El estudio serológico para los virus hepatitis A, B y $\mathrm{C}$ resultó negativo. Además se realizó un hemocultivo (al ingreso). Se inició terapia antibacteriana empírica intravenosa con ceftriaxona $2 \mathrm{~g}$ al día y metronidazol 500 $\mathrm{mg}$ fraccionado cada $8 \mathrm{~h}$. A las $15 \mathrm{~h}$ de incubación, hubo desarrollo de bacilos gramnegativos en el hemocultivo, identificado como $V$. cholerae mediante espectrometría de masas MALDI-TOF MS (Vitek MS, bioMérieux, Marcy l' Etoile, France) (Figura 1). La cepa se envió al laboratorio de referencia nacional (Instituto de Salud 

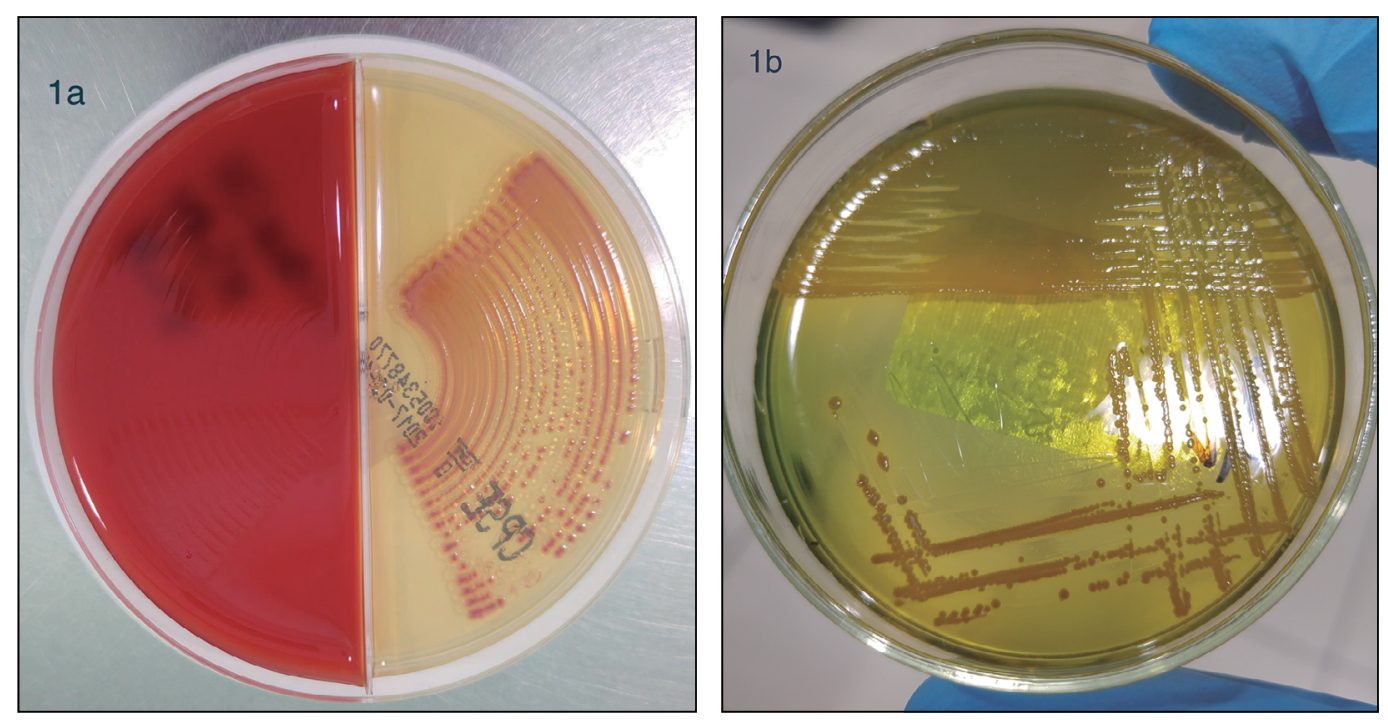

Figura 1. Colonias de $V$. cholereae no-O1/ no-0139 en medio CPSE (a) y TCBS (b) incubadas a $37^{\circ}$. Se aprecian colonias sacarosa positiva.

Pública de Chile) (ISP), de acuerdo a la norma local de vigilancia de enteropatógenos. La paciente evolucionó favorablemente, por lo que se decidió cambiar la terapia a doxiciclina $100 \mathrm{mg}$ cada $12 \mathrm{~h}$ hasta completar siete días de tratamiento en total. Hubo regresión completa de los síntomas y normalización de las pruebas hepáticas.

\section{Caracterización molecular}

El ISP confirmó la identificación informando una cepa no toxigénica no-O1/no-139. El estudio de los factores de virulencia se realizó en el Programa de Microbiología del Instituto de Ciencias Biomédicas (ICBM) de la Universidad de Chile.

Los partidores utilizados en la caracterización molecular se muestran en la Tabla 1. La cepa mostró ausencia de genes de virulencia clásicos como la toxina colérica (gen $c t x A$ ) y el pilus TCP (gen $t c p A$ ). Sin embargo, se observó la presencia de cinco de los seis genes de virulencia correspondientes a una isla de patogenicidad homóloga a la denominada VPaI-7 de Vibrio parahaemolyticus (vcsN2 ,$+ v c s C 2+, v c s V 2+$, tox $R$-, vsp $D+, v o p F+$ ). Además, fue positiva para los genes de virulencia propios de $V$. parahaemolyticus como hylA y rtx $A$, pero que se encuentran fuera de la isla.

\section{Discusión}

Se presenta el caso de una paciente chilena con una bacteriemia por una cepa de $V$. cholerae no-O1/no-O139 portadora de un segmento homólogo de la isla de patogenicidad denominada VPaI-7 de V. parahaemolyticus. En Chile, desde 1998 que no se registran nuevos casos autóctonos de cepas toxigénicas (O1 y O139) ${ }^{11}$. Por el contrario, este es el tercer caso reportado de infección por $V$. cholerae no-O1/no-O139 en los últimos 10 años en nuestro país ${ }^{7,12}$. Estas cepas no toxigénicas forman parte de los ecosistemas acuáticos principalmente en zonas costeras tales como estuarios y aguas de baja salinidad junto a otras especies de Vibrio spp. ${ }^{13}$. Su

Tabla 1. Partidores diseñados para este estudio y utilizados en la tipificación de cepas chilenas de origen clínico de $V$. cholerae no-01/no-0139

\begin{tabular}{|c|c|c|}
\hline Partidores & Secuencia & Tamaño (pb) \\
\hline VCNN-N2f & $5^{\prime}$-CAA TCG CAG GAT AAC GTC CT-3' & 874 \\
\hline VCNN-N2r & $5^{\prime}$-CGA GGC GAA ATT GTC AAA GT-3' & \\
\hline VCNN-C2f & 5'-CTC GAG GAG GGT TAA TGT CG-3' & 1.050 \\
\hline VCNN-C2r & 5'-GCG CAG GCA GAT GTT TTT AT-3' & \\
\hline VCNN-V2f & $5^{\prime}$-ATT GCA CAA GTA GCC GCT TT-3' & 1.438 \\
\hline VCNN-V2r & $5^{\prime}$-GAA ACC GTC GGT CAG TTT GT-3' & \\
\hline VCNN-VopFf & $5^{\prime}$-CTG CCG TCA ATT TGG AAG AT-3' & 1.265 \\
\hline VCNN-VopFr & $5^{\prime}$-AAG TGC TGC CAA TTG AGC TT-3' & \\
\hline VCNN-VspDf & 5'-CTT GCT CAA TTC GTG TTT GC - -3' & 853 \\
\hline VCNN-VspDr & $5^{\prime}$-AAC CAA CCT CAG CAA CAA GC-3' & \\
\hline VCNN-ToxR2f & $5^{\prime}$-ACA GGA GCC AAC TGG GTT TA-3' & 369 \\
\hline VCNN-ToxR2r & 5' -TGC TGT GTG AAA ATG CCA AT-3' & \\
\hline VCNN-HylAf & $5^{\prime}$-AGA TCA ACT ACG ATC AAG CC-3' & 1.677 \\
\hline VCNN-HylAr & $5^{\prime}$-AGA GGT TGC TAT GCT TTC TAC-3' & \\
\hline VCNN-RtxAf & $5^{\prime}$-GCG ATT CTC AAA GAG ATG C-3' & 1.366 \\
\hline VCNN-RtxAf & $5^{\prime}$-CAC TCA TTC CGA TAA CCA C-3' & \\
\hline
\end{tabular}


transmisión se ha asociado al consumo de alimentos de origen marino, especialmente bivalvos crudos que podrían estar colonizados en un alto porcentaje $(5,6 \%$ a $32 \%)^{13,14}$. En nuestro país, se ha observado la presencia de cepas no-O1/no-O139 en 6,6\% de muestras de agua (servidas, alcantarillado, canales de regadío) y en $0,3 \%$ de alimentos (hortalizas y moluscos) ${ }^{15}$, los cuales representan potenciales fuentes de infección. Al igual que en 50 a $75 \%$ de los $\operatorname{casos}^{9}$, no se logró identificar la fuente de infección.

La identificación del género y la especie se realizó mediante MALDI-TOF MS, el cual ha sido validado para la identificación de especies del género Vibrio, demostrando excelente concordancia con los métodos de biología molecular ${ }^{16}$. La caracterización genética mediante RPC reveló ausencia de la toxina colérica y del pilus TCP, lo que sugiere la existencia de otros factores de virulencia en este tipo de cepas. Los genes presentes en la isla de patogenicidad homóloga (VpaI-7) de $V$. parahaemolyticus codifican para un sistema de secreción tipo III (T3SS), un organelo especializado en forma de "jeringa molecular", que permite translocar toxinas y otras proteínas efectoras desde la bacteria al interior de la célula hospedera, permitiendo la manipulación de sus funciones y generando citotoxicidad ${ }^{17-19}$. Estudios previos de cepas ambientales han comunicado la presencia de algunos de estos genes en baja frecuencia ${ }^{20}$. Previamente, Ulloa y cols. reportaron el caso de una mujer hospitalizada por gastroenteritis, en la que se identificó la presencia de $V$. cholerae no-O1/no-139 portador de un trozo de la isla de patogenicidad VpaI-7 en muestra de heces ${ }^{12}$. Ambos casos sugieren la transferencia horizontal de genes de $V$. parahaemolyticus hacia cepas no toxigénicas de $V$. cholerae, los cuales probablemente contribuyen en la patogenicidad para el ser humano ${ }^{12}$. Un estudio experimental demostró que conejos inoculados con cepas portadoras de esta isla desarrollaban cuadros de diarrea, fiebre y desenlace fatal. Posteriormente, se comprobaron alteraciones morfológicas en la pared intestinal. En cambio, cuando se les inoculaban cepas con mutación del TSS3 (inactivándolo), los conejos no presentaban enfermedad clínica ${ }^{17}$.

La expansión en la portación de esta isla de patogenicidad en cepas "no toxigénicas" podría tener un impacto directo en la salud de la población, aumentando los casos de enfermedad. Entre el año 2015 y 2017 se confirmaron sólo 15 casos de Vibrio cholerae no-O1/ no-O139, todos en forma esporádica. Sin embargo, desde el segundo semestre de 2018 se ha observado en Chile un aumento inesperado de estos casos, reportándose a la fecha 70 casos sospechosos ( 55 confirmados). Si bien no se han registrado casos fallecidos, hubo 13 pacientes que requirieron hospitalización (Ministerio de Salud, datos no publicados $)^{21}$. Esto ha motivado a reforzar los sistemas de vigilancia epidemiológica, el estudio de muestras ambientales provenientes de agua y alimentos, y la difusión en las medidas de prevención ${ }^{11}$.

Además, la cepa estudiada fue positiva para hylA y $\operatorname{rtx} A$, siendo ambos reconocidos genes de virulencia ubicados fuera de la isla y que han sido identificados en cepas ambientales de $V$. cholerae $e^{22-23}$ y en cepas de pacientes con infecciones gastro-intestinale ${ }^{14}$ y extraintestinales que carecían del $\mathrm{T}_{3 \mathrm{SS}}{ }^{10}$. Específicamente, el gen $r t x A$ codifica para un complejo de polipéptidos de alto peso molecular que contiene toxinas multifuncionales. Tras ser liberado, éste es capaz de auto-procesarse en la membrana de la célula diana, permitiendo que sus efectores ingresen a la célula y alteren el ensamblaje y la señalización de la actina del citoesqueleto. De esta forma, se ven afectadas las uniones intercelulares, el transporte celular interno y la fagocitosis. A diferencia del T3SS no se requiere un contacto directo con la célula diana para generar el daño ${ }^{24,25}$.

Finalmente, se ha descrito una letalidad aproximada de $30 \%$ en los casos de bacteriemia por $V$. cholerae no-O1/no-139. Algunos factores asociados a mortalidad son la cirrosis hepática, las neoplasias malignas y el uso de corticoesteroides ${ }^{9,26}$. El diagnóstico tardío y la terapia inadecuada o incompleta son otros factores pronósticos relacionados 9 . En este caso en particular, la ausencia de los factores de riesgo mencionados y el tratamiento oportuno posiblemente fueron determinantes para la favorable evolución observada.

\section{Conclusión}

Se reporta el primer caso publicado en Chile de bacteriemia por $V$. cholerae no-O1, no O-139 que porta una región homóloga a la isla de patogenicidad VpaI-7 del genoma del $V$. parahaemolyticus que codifica para un sistema de secreción tipo III (T3SS).

\section{Resumen}

Presentamos un caso de bacteriemia por Vibrio cholerae no-O1/ no-O139 en una mujer de 81 años con un cuadro de dolor abdominal, fiebre, vómitos, diarrea, coluria e ictericia, mientras visitaba una zona rural sin acceso a agua potable. La identificación se realizó por la técnica de espectrometría de masa MALDI-TOF, confirmándose una cepa no toxigénica no-O1/no-139. La caracterización molecular del aislado demostró la ausencia del gen de la toxina del cólera (CTX), y pilus TCP; sin embargo, presentó cinco de los seis genes de virulencia presentes en la isla de patogenicidad 
homóloga denominada VPaI-7 del $V$. parahaemolyticus (vcs $\mathrm{N} 2+, v c s \mathrm{C} 2+, v c s \mathrm{~V} 2+$,toxR-, vsp $\mathrm{D}+$, $\mathrm{T} v o p \mathrm{~F}+$ ). Además, el aislado presentó los genes de virulencia hylA y rtxA. Este es el primer caso reportado en Chile de una cepa clínica de $V$. cholerae no-O1, no-O139 aislada de hemocultivos portador de un segmento homólogo de la isla de patogenicidad denominada VPaI-7 de $V$. parahaemolyticus, el cual codifica para un sistema de secreción tipo III (TTSS), que probablemente contribuye a su virulencia.

\section{Referencias bibliográficas}

1.- Devault A M, Golding G B, Waglechner N, Enk J M, Kuch M, Tien J H, et al. Second-pandemic strain of Vibrio cholerae from the Philadelphia cholera outbreak of 1849. N Engl J Med 2014; 370: 334-40. doi: 10.1056/NEJMoa1308663.

2.- Cholera Working Group. Large epidemic of cholera-like disease in Bangladesh caused by Vibrio cholerae O139 synonym Bengal. Lancet 1993; 342: 387-90.

3.- Johnson J A, Salles C A, Panigrahi P, Albert M J, Wright A C, Johnson R J, et al. Vibrio cholerae $\mathrm{O} 139$ synonym bengal is closely related to Vibrio cholerae El Tor but has important differences. Infect Immun 1994; 62: 2108-10.

4.- Chin C S, Sorenson J, Harris J B, Robins W P, Charles R C, Jean-Charles R R, et al. The origin of the Haitian cholera outbreak strain. N Engl J Med 2011; 364: 33-42.

5.- Faruque S M, Albert M J, Mekalanos J J. Epidemiology, genetics, and ecology of toxigenic Vibrio cholerae. Microbiol Mol Biol Rev 1998; 62: 1301-14.

6.- Waldor M, Ryan E, Chapter 216: Vibrio cholerae. Bennett JE, Dolin R, Blaser MJ, editors. Mandell, Douglas and Bennett's Principles and Practice of Infectous Diseases, 8th ed. Philadelphia: Elsevier; 2015 , p. 2471-9.

7.- Briceño L I, Puebla A C, Guerra A F, Jensen F D, Núñez B H, Ulloa F MT, et al. Septicemia fatal causada por Vibrio cholerae no-O1, no-O139 en Chile. Caso clínico. Rev Med Chile 2009; 137: 1193-6. doi: /S003498872009000900008.

8.- Trubiano J A, Lee J Y, Valcanis M, Gregory J, Sutton BA y Holmes NE. Non-O1, non-O139 Vibrio cholerae bacteraemia in an Australian population. Intern Med J 2014; 44: 508-11. doi: 10.1111/imj.12409.

9.- Deshayes S, Daurel C, Cattoir V, Parienti J J, Quilici M L, de La Blanchardiere A. Non-O1, non O-139 Vibrio cholerae bacteraemia: case report and literature review. Springerplus 2015; 4: 575. doi: 10.1186/s40064-015-1346-3

10.- Chowdhury G, Joshi S, Bhattacharya S, Sekar U, Birajdar B, Bhattacharyya A, et al. Extraintestinal infections caused by non- toxigenic Vibrio cholerae non-O1/non-O139. Front Microbiol 2016; 7: 144. doi: 10.3389/ fmicb.2016.00144

11.- Ministerio de Salud de Chile, Departamento de Epidemiología. Situación epidemiológica de cólera en Chile (Informe año 2017). Disponible en: http://epi.minsal.cl/colera-materialesrelacionados/ (Fecha de acceso: 15 de mayo de 2019).

12.- Ulloa M T, Porte L, Braun S, Dabanch J, Fica A, Henríquez T, et al. Gastroenteritis aguda causada por Vibrio cholerae no-O1, no O-139 que porta un región homóloga a la isla de patogenicidad Vpal-7. Rev Chilena Infectol 2011; 28: 470-73. doi: /S071610182011000600012 .

13.- Huehn S, Eichhorn C, Urmersbach S, Breidenbach J, Bechlars S, Bier N, et al. Pathogenic vibrios in evironmental, seafood and clinical sources in Germany. Int J Med Microbiol 2014; 304: 843-50. doi: 10.1016/j. ijmm.2014.07.010.

14.- Ottaviani D, Leoni F, Rocchegiani E, Santarelli S, Masini L, Di Trani V, et al. Prevalence and virulence properties of non-O1 non-O139 Vibrio cholerae strains from seafood and clinical samples collected in Italy. Int J Food Microbiol 2009; 132: 47-53. doi: 10.1016/j. ijfoodmicro.2009.03.014.

15.- Ministerio de Salud de Chile, Instituto de Salud Pública. Informe de resultados de vigilancia de laboratorio Vibrio cholerae en muestras ambientales 2010-2012. Disponible en: http:// www.ispch.cl/documento/18683 (Fecha de acceso: 15 de mayo de 2019).

16.- Erler R, Wichels A, Heinemeyer E A, Hauk G, Hippelein M, Reyes N T, et al. VibrioBase: A MALDI-TOF MS database for fast identification of Vibrio spp. that are potentially pathogenic in humans. Syst Appl Microbiol 2015; 38: 16-25. doi: 10.1016/j. syapm.2014.10.009.

17.- Shin O, Tam V, Suzuki M, Ritchie J M, Bronson R T, Waldor M K, et al. Type III secretion is essential for the rapidly fatal diarrheal disease caused by non-O1, non-O139 Vibrio cholerae. MBio 2011; 2: e00106-11. doi: 10.1128/mBio.00106-11.

18.- Ham H, Orth K. The role of type III secretion system 2 in Vibrio parahaemolyticus pathogenicity. J Microbiol 2012; 50: 719-25. doi: 10.1007/s12275-012-2550-2.

19.- Calder T, de Souza Santos M, Attah V, Klimko J, Fernández J, Salomon D, et al. Structural and regulatory mutations in Vibrio parahaemolyticus type III secretion systems display variable effects on virulence. FEMS Microbiol Lett 2014; 361: 107-14. doi: 10.1111/1574-6968.12619.

20.- Ceccarelli D, Chen A, Hasan N A, Rashed S M, Hug A, Colwell R R. Non-O1/non-O139 Vibrio cholerae carrying multiple virulence factors and V. cholerae $\mathrm{O} 1$ in the Chesapeake Bay, Maryland. Appl Environ Microbiol 2015; 81: doi: 10.1128/AEM.03540-14.

21.- Ministerio de Salud de Chile, Departamento de Epidemiología. Minuta: Situación epidemiológica de brote de diarrea aguda por Vibrio cholerae no toxigénico en Chile, año 2018-2019. Actualizada: 11 de marzo 2019 (no publicado).

22.- Rajpara N, Vinothkumar K, Mohanty P, Singh A K, Singh R, Sinha R, et al. Synergistic effect of various virulence factors leading to high toxicity of environmental $V$. cholerae Non-O1/non-O139 isolates lacking ctx gene : comparative study with clinical strains. PLoS One 2013; 8: e76200. doi: 10.1371/journal. pone. 0076200 .

23.- Siriphap A, Leekitcharoenphon P, Kaas R S, Theethakaew C, Aarestrup F M, Sutheinkul O, et al. Characterization and genetic variation of Vibrio cholerae isolated from clinical and environmental sources in Thailand. PLoS One 2017; 12: e0169324. doi: 10.1371/journal. pone. 0169324.

24.- Dolores J, Satchell K J. Analysis of Vibrio cholerae genome sequences reveals unique rtxA variants in environmental strains and an rtxA-null mutation in recent altered El Tor isolates. MBio 2013; 4: e00624-12. doi: 10.1128/mBio.00624-12.

25.- Satchell K J F. Multifunctional-autoprocessing repeats-in-toxin (MARTX) Toxins of Vibrios. Microbiol Spectr 2015; 3(3). doi: 10.1128/ microbiolspec.VE-0002-2014.

26.- Ou T Y, Liu J W, Leu H S. Independent prognostic factors for fatality in patients with invasive Vibrio cholerae non-O1 infections. J Microbiol Immunol Infect 2003; 36: 117-22. 